\title{
Automatische Erstellung von digitalen Simulationszwillingen von Produktionssystemen
}

\author{
Walter Wincheringer, Tobias Sohny, Marec Kexel \\ Digitales Produktionslabor, Hochschule Koblenz, Konrad-Zuse-Straße 1, 56075 Koblenz, Germany; \\ dpl@hs-koblenz.de
}

\begin{abstract}
Die durch die Industrie 4.0 postulierte Mass Customization führt zu einer hohen Planungskomplexität einer Produktion, was den Einsatz von diskreten Simulationssystemen erfordert. Die Erstellung eines digitalen Simulationszwillings ist jedoch zeitaufwändig, insbesondere, wenn sich in einer diskreten Werkstatt-, Matrixfertigung die Anforderungen häufig ändern. In diesem Beitrag wird ein Ansatz aufgezeigt, welcher die Generierung ablauffähiger Simulationsmodelle von Produktionssystemen, über eine VBASchnittstelle und ohne Programmierarbeit durch den Anwender, ermöglicht. Die Wahl verschiedener Prioritätsregeln zur Ablaufplanung, in Abhängigkeit typischer Produktionsziele, und deren Simulation wurden entwickelt. Somit sind Produktionsplaner in der Lage ihre Planung durch Simulation in kürzester Zeit abzusichern.
\end{abstract}

\section{Einleitung}

Durch die Globalisierung, kürzere Produktlebenszyklen sowie die Individualisierung der Kundenwünsche sind Unternehmen in ihrer Wettbewerbsfähigkeit gefordert. Um den Anforderungen gerecht zu werden, haben viele Unternehmen in den letzten Jahren ihr Produktionssystem nach Lean-Gesichtspunkten reorganisiert [1]. Mit Hilfe der Lean-Management-Methoden oder gemäß den Gestaltungsrichtlinien ganzheitlicher Produktionssysteme ist es gelungen die gestiegene Produktvielfalt zu beherrschen. Die Mass Customization schreitet jedoch weiter voran und bedingt, in Verbindung mit dynamischen Märkten, eine hohe Flexibilität und eine Wandelbarkeit des Produktionssystems. Dies führt zu einer zunehmenden Komplexität in der Produktionsplanung und -steuerung [2].

Das Konzept von Industrie 4.0 postuliert eine variantenreiche Produktion mit Losgröße Eins zu den Kosten einer Serienfertigung. Daher werden sich die klassischen Fertigungsprinzipien nach dem Flussprinzip, Reihen- und Fließfertigungen, in Richtung einer Matrixproduktion wandeln. Hierzu werden flexible Produktionskapazitäten zukünftig nicht mehr mit Förderbändern statisch verknüpft, sondern über fahrerlose Transportsysteme dynamisch miteinander verbunden. Dadurch entstehen wandlungsfähige Produktionsabläufe mit unterschiedlichen Produktionstopologien die nach dem "plug and produce"-Konzept bedarfsgerecht, in Abhängigkeit der kundenspezifischen Auftragsstruktur, stets neu konfiguriert werden können. [2]

Auch im modernsten Produktionssystem müssen die klassischen Aufgaben der Produktionsplanung, der Kapazitätsauslastung sowie die Durchlaufterminierung, in Abhängigkeit vom jeweiligen Auftragsbestand, ausgeführt werden. Die Wandlungsfähigkeit der Produktion führt hierbei zu einer Planungskomplexität, die ohne den Einsatz digitaler Werkzeuge nicht beherrschbar ist.

Die ereignisdiskrete Simulation (discrete event simulation, DES) ist hierzu ein anerkanntes Werkzeug. Die Nachbildung existenter oder geplanter Systeme in ablauffähigen Simulationsmodellen, erlauben die Abbildung der bestehenden Dynamik [3].

Dieses Simulationsmodell, als digitaler Zwilling (Digital Twin, DT) der Produktionstopologie, muss mit der notwendigen Genauigkeit an das geplante oder bestehende Produktionssystem angepasst werden [4]. Dazu ist eine regelmäßige Anpassung des DT bzw. dessen Neuerstellung (bei Anpassung der Produktionsstruktur) erforderlich.

Der Aufwand zur Anpassung oder Neuerstellung von Simulationsmodellen ist jedoch zeitaufwendig, insbesondere, wenn verschiedene Produktionstopologien wöchentlich überprüft werden müssen [5]. Somit ist bei der Erstellung eines DT ein Ansatz zu wählen, welcher einen reduzierten Modellierungsaufwand begünstigt und eine Rekonfiguration des digitalen Abbilds zulässt. 


\section{Stand der Technik}

Matrixfertigung. Mittels der Matrixfertigung lassen sich kundenindividuelle Produkte in der nötigen Flexibilität und Losgröße Eins produzieren. Eine Matrixfertigung besteht u.a. aus einer Anzahl an Produktionsprozessen (Betriebsmittel), mit jeweils spezifischen Leistungsprofilen bzw. den Fähigkeiten, bestimmte Prozessschritte durchführen zu können. Auftragsspezifische Arbeitsvorgänge können infolgedessen durch einen oder mehrere Produktionsprozesse bearbeitet werden. Der Transport zwischen den Ressourcen erfolgt auftragsspezifisch und flexibel, unter Verwendung manueller oder autonomer Transportvorrichtungen, sog. fahrerlosen Transportsystemen (FTS). Der Maschinenbediener, als weitere wichtige Ressource eines Produktionssystems, wird bzgl. Qualität (Tätigkeitsprofil) und Quantität als ausreichend verfügbar angenommen [6].

Erste Beispiele sind bereits in der Roboterfertigung [7] oder auch in der PKW-Montage [8] realisiert oder befinden sich in der Planung [9].

FJSSP. Das sich daraus entwickelnde Produktionsablaufplanungsproblem, wird in der Literatur als „Flexible Job Shop Scheduling Problem“ bezeichnet [10]. Dies stellt, durch den komplexen Materialfluss, ein kombinatorisches Problem dar, dessen Optimum nicht durch analytische Berechnungen ermittelt werden kann. Die Anzahl der möglichen Reihenfolgen kann dabei, in Abhängigkeit der Ressourcenanzahl m, der Auftragsanzahl $n$ und der Flexibilität, bis zu $(n !)^{(m)}$ mögliche Maschinenbelegungen ergeben. Dabei muss neben der Zielgröße Termintreue auch die Durchlaufzeit (Bestände) und die Betriebsmittelauslastung berücksichtigt werden [11]. Die Generierung einer vermeintlich optimalen Maschinenbelegung führt innerhalb klassischer PPS Systeme zu einem hohen Planungs- und Rechenaufwand. Hierbei erfolgt die Terminierung der Ressourcennutzung häufig durch starre Verfahren bei fehlender Transparenz über die im Hintergrund laufende Prozesskoordination des PPS-Systems. Der Produktionsplaner bedient das PPS-System ohne die Möglichkeit einer Plausibilitätsüberprüfung der durch das System vorgeschlagenen optimalen Lösung, da hinterlegte Lösungsalgorithmen nicht bekannt sind oder deren Berechnung nicht beeinflusst werden kann [12].

Durch die hohe Relevanz wurden in den letzten Jahren hierzu mehrere Methoden entwickelt. Diese lassen sich in exakte Bewertungsverfahren, welche aufgrund einer hohen Rechenleistung nicht weiter betrachtet werden, sowie heuristische Ansätze differenzieren (Abbildung 1) [13].

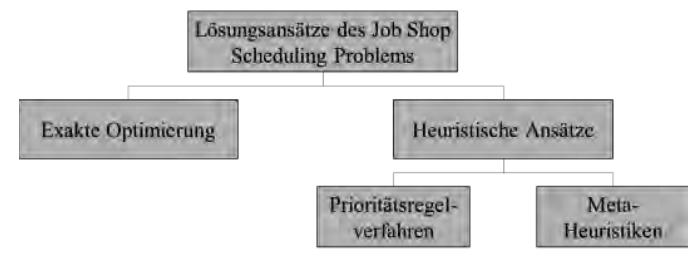

Abbildung 1: Lösungsansätze des Job Shop Scheduling Problems

Heuristische Ansätze stellen Näherungsverfahren dar, die zwar keine optimale Lösung garantieren, jedoch bei begrenzter Rechenleistung gute und zulässige Resultate ermöglichen. Hierzu zählen die sogenannten Prioritätsregelverfahren [14]. Die Meta-Heuristiken sind iterative Verfahren, welche ausgehend von einer Startsituation schrittweise Optimierungen berechnen, unter Berücksichtigung von Lerneffekten, was einen nicht unerheblichen Aufwand zur Programmierung bedingt [13].

Der Einsatz von Prioritätsregeln eignet sich somit aufgrund des geringen Rechenaufwands, einer Komplexitätsunabhängigkeit sowie einer einfachen Implementierung und schnellen Anpassung.

Zu den Prioritätsregeln zählen u.a.:

- FIFO (First in Firs Out)

- SPT (Shortest Processing Time)

- EDD (Earliest Due Date)

- LWKR (Least Work Remaining)

Im Kontext der PPS bedingt dies eine sinnvolle Zuordnung der Arbeitsgänge zu den entsprechenden Ressourcen, als auch eine sinnvolle Abarbeitungsreihenfolge, mit dem Ziel eine möglichst geringe Durchlaufzeit (DLZ) und eine hohe Auslastung der Betriebsmittel (BM), zu realisieren.

Mit Hilfe der Prioritätsregelverfahren kann eine Lösung, jedoch keine optimale Lösung, für das Planungsproblem erstellt werden. Hinzu kommt, dass unterschiedliche Prioritätsregeln und unterschiedliche Kombinationen zur Lösung eingesetzt werden können. Ob die Lösung jedoch die Ziele der Produktionsplanung (u.a. Termintreue, Kapazitätsauslastung) tatsächlich erfüllt, ist erst nach Ablauf der Produktion - im Nachhinein - fest- 
stellbar. Darüber hinaus ist die Produktion durch unterschiedliche Ereignisse, wie z.B. Eilaufträge, Qualitätsprobleme, technische oder organisatorische Störungen, so dynamisch, dass es häufig zu erheblichen Abweichungen vom Planungsergebnis kommt [15]. Um die dynamischen Ereignisse über die Zeit in Produktionsprozessen zu berücksichtigen, hat sich die diskrete Event-Simulation bewährt [13].

Simulation. In Produktion und Logistik wird Simulation definiert als das "Nachbilden eines Systems mit seinen dynamischen Prozessen in einem experimentierbaren Modell, um zu Erkenntnissen zu gelangen, die auf die Wirklichkeit übertragbar sind; insbesondere werden die Prozesse über die Zeit entwickelt" [3]. Diese ausführbaren Modelle (Simulationsmodelle) ermöglichen eine Analyse des Systemverhaltens nach Parameter- oder Strukturänderungen.

Für die Erstellung von Simulationsmodellen ist ein genaues Verständnis des Systems sowie die Programmierung des Simulationsmodells erforderlich. Dies ist zeitaufwändig und ressourcenintensiv.

Bzgl. einer Matrixfertigung ist die vom Produktionsplaner getroffene Produktionstopologie als Simulationsmodell des Produktionssystems zu erstellen, um die, in Abhängigkeit der gewählten Prioritätsregeln, entstehende Ablaufplanung abzusichern. Bedingt dadurch, dass die Produktionstopologie häufig angepasst werden muss, ist eine stetige Neuerstellung eines Modells zu aufwendig und wirtschaftlich nicht tragbar.

Eine aufwandsarme Simulationsmodellerstellung, sowie die Wiederverwendung bereits erstellter Modelle, verspricht die Generierung. Dieser Ansatz gliedert sich in drei Aufgabenbereiche: die Generierung, die Initialisierung und die Adaption des Simulationsmodells [4].

Generierung. Die Generierung beschreibt die eigentliche Erzeugung des Simulationsmodells inklusive der Hinterlegung entsprechender Parameter (Bsp.: produktspezifische Zyklus-, Rüstzeiten, Steuerungsregeln, etc.) [4].

Initialisierung. Im Anschluss an die Generierung ist eine Initialisierung des Simulationsmodells nötig, um eine entsprechende Ausgangssituationen zum Start der Simulation festzulegen (Bsp.: Bearbeitungsstände, Betriebsmittelzustände, etc.) [16].
Adaption. Die Adaption beinhaltet das Ändern des generierten Simulationsmodells, ohne eine vollständige Neugenerierung des Simulationsmodells vorzunehmen (Bsp.: Veränderung der Betriebsmittelkapazitäten, der Losgrößen, etc.) [17].

Die genannten Aufgabenbereiche ermöglichen somit das automatische Erstellen und Anpassen eines Simulationsmodells für den Produktionsablauf, in Abhängigkeit der vom Produktionsplaner gewählten Produktionstopologie, inkl. einer produktspezifischen Parametrierung der Betriebsmittel, die Zuordnung von Logiken sowie die Initialisierung einer Ausgangssituation der Produktion. [4].

\section{Umsetzung}

Für die Realisierung eines Simulationsmodell-Generators, der eine automatische Erstellung eines Produktionssimulationsmodells ermöglicht, sind zunächst die Produktionsprozesse (Betriebsmittel), Materialpuffer, Transportvorrichtungen, FTS, etc. und die Prioritätsregeln als Module abzubilden.

Die einzelnen Module repräsentieren hierbei einzelne abgeschlossene und parametrierbare Teilsysteme, welche eine Mehrfachverwendung sowie deren Aufruf - in der Simulationsumgebung - ermöglichen. Zudem lassen sich die einzelnen Module an den erforderlichen Schnittstellen miteinander verknüpfen.

Die einzelnen Betriebsmittel (Ressourcen) sind in der Simulatorumgebung WITNESS bereits als einzelne Module vorhanden und können für den Ansatz der Generierung verwendet werden. Für eine hinreichend genaue Abbildung bedarf es der Angaben zur technischen und organisatorischen Verfügbarkeit, Qualitätsraten und Rüstzeiten, inklusive ihrer jeweils spezifischen Verteilungen. Diese erforderlichen Daten lassen sich mittels einer VBA-Schnittstelle durch eine vereinfachte Eingabeoberfläche hinterlegen (Abbildung 2).

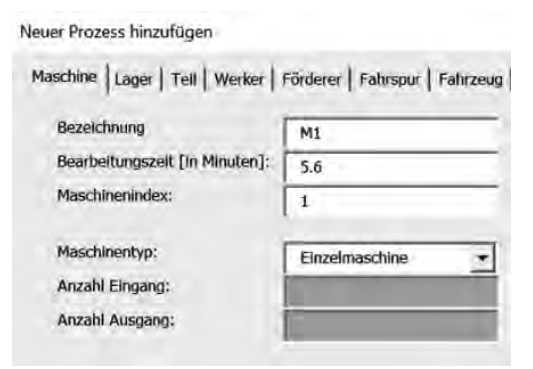

Abbildung 2: Ausschnitt aus der Anwenderoberfläche 
Daten über die Verfügbarkeit von Werkzeugen, Rohmaterial und Mitarbeiter werden im Simulationsmodell als vorhanden angenommen. Als Datenquelle können historische Produktions- und Auftragsdaten, z.B. aus einem MES, MDE, BDE, entnommen, aufbereitet und zur Anwendung kommen.

Die einzelnen Betriebsmittel lassen sich mittels dieser Schnittstelle parametrisieren. Dem Anwender wird deren Anordnung im Fabriklayout mittels eines Koordinatensystems ermöglicht, sodass ein visueller Bezug zum Realsystem besteht (Abbildung 3).

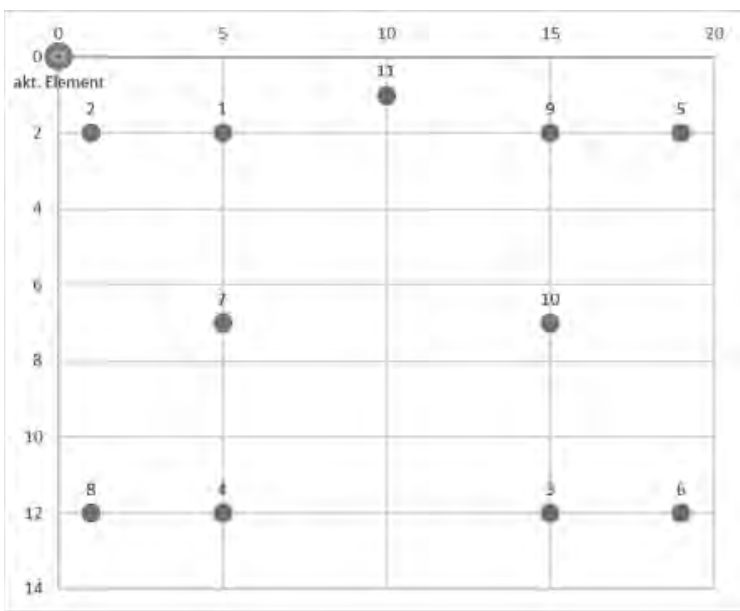

Abbildung 3: Vereinfachte Layout-Darstellung in der Anwenderoberfläche

Mittels einer Transportmatrix, welche die quellenund senkenspezifische Distanz und die damit einhergehende Transportzeit inkl. der Be- und Entladezeit berücksichtigt, ist die Möglichkeit den Materialfluss mit realitätsnahen Prozesszeiten abzubilden, gegeben.

Zur Realisierung dieses Materialflusses lassen sich die simulatorspezifischen Regeln (Push/Pull) verwenden. Diese erlauben es einfache Flüsse, von Quellen zu Senken, inklusive der entsprechenden Steuerungslogik abzubilden. Zur Abbildung einer Matrixfertigung ist die Verknüpfung des Materialflusses allerdings von den Aufträgen, deren spezifischen Arbeitsvorgängen und den geplanten Abläufen, welche mit Hilfe der gewählten Prioritätsregeln geplant werden, abhängig. Hierzu sind alle Betriebsmittel-Module, inkl. Puffer-Module, mittels FTS automatisch untereinander verknüpft, die logische Reihenfolge wird mittels Prioritätsregel-Module realisiert.

Die jeweilige Zykluszeit der Produktionsprozesse ist spezifisch, je nachdem um welche Maschine oder Auftrag bzw. Arbeitsvorgang es sich handelt, zu hinterlegen.
Hierzu kann mittels der VBA-Schnittstelle die Anzahl der Betriebsmittel definiert werden. Darüber hinaus erlaubt diese den fertigungspezifischen Ablauf eines Auftrags inkl. der Betriebsmittel zu hinterlegen (Tabelle 1)

\begin{tabular}{|c|c|c|c|c|c|c|c|c|}
\hline \multicolumn{10}{|c|}{ Arbeitsplan Fallbeispiel } \\
\hline $\mathrm{j}$ & M1 & M2 & M3 & M4 & M5 & M6 & M7 & M8 \\
\hline 1 & 1 & 1 & 3 & 3 & 2 & 4 & 2 & 5 \\
\hline 2 & 1 & 4 & 3 & 5 & 2 & 2 & & 6 \\
\hline 3 & 1 & 4 & 3 & 5 & 2 & 7 & 2 & 6 \\
\hline 4 & 1 & 1 & 3 & 3 & 2 & 4 & 2 & 4 \\
\hline 5 & 3 & 1 & 3 & 1 & 2 & 4 & 2 & 4 \\
\hline \multicolumn{10}{|c|}{$\mathrm{j}=$ Auftrag } & \multicolumn{7}{|c|}{ Maschine } \\
\hline
\end{tabular}

Tabelle 1: Exemplarischer Arbeitsplan

Dieser fertigungsspezifische Ablauf ist dem jeweiligen Auftrag j mittels Attribute zugeordnet, sodass alle Abläufe in der richtigen Reihenfolge berücksichtigt werden. Die Zuordnung zu den jeweiligen Ressourcen (M18) sowie der Anordnung in deren Warteschlangen ist abhängig von den gewählten Prioritätsregeln.

Die Prioritätsregeln wurden simulatorspezifisch in einzelne Logik-Module codiert. Dies bedingt, einen einmaligen Codieraufwand. Eine Kombination, Mehrfachverwendung sowie eine Erweiterung der Prioritätsregeln werden hierdurch ermöglicht. Die jeweilige Anzahl der Pufferplätze vor den jeweiligen Betriebsmitteln, lassen sich zudem individuell einstellen. Gleiches gilt für die Anzahl der FTS und ihrer Transportkapazität.

Die Abarbeitung der Transportaufträge erfolgt hierbei nach dem FIFO-Prinzip. Ein Transportauftrag wird beim Start des Fertigungsauftrags, für den erster Arbeitsvorgang, sowie nach Fertigstellung am jeweiligen Produktionsprozess generiert.

Alle Daten werden in einem für den Generator geeigneten Format gespeichert. Beim Start der Simulation, wird automatisch ein lauffähiges Simulationsmodell, anhand der hinterlegten Daten, generiert.

Unmittelbar nach der Generierung des Simulationsmodells, wird eine Initialisierungsdatei geladen. Die dort hinterlegten Informationen aus der VBA-Schnittstelle, erlauben es die aktuelle Situation der Produktion abzubilden. So lassen sich beispielweise die Bearbeitungsfortschritte von Restaufträgen, inkl. der vorhandenen Bestände in der Produktion (WIP), hinterlegen. Auch diese Daten können aus einem MES oder BDE-System übernommen werden. Nach der Initialisierung startet der Simulationslauf (Abbildung 4). 


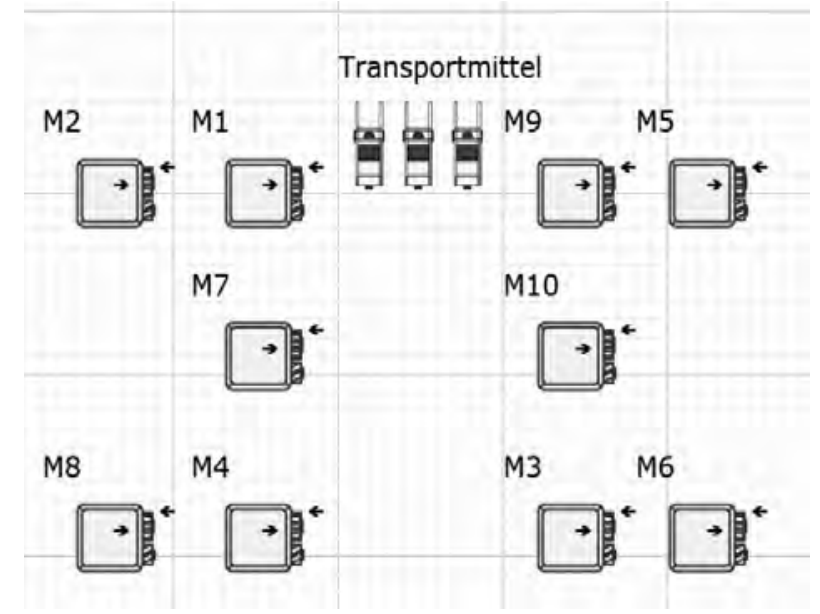

Abbildung 4: Generiertes und lauffähiges Simulationsmodell

Eine anschließende Adaption des generierten Simulationsmodells lässt sich effizient in der VBA-Schnittstelle einpflegen, wodurch Betriebsmittel hinzugefügt oder geändert werden können und die Realität - abhängig von der aktuellen Planungssituation - hinreichend genau abgebildet werden kann.

Für den Fall, dass die KPIs, z.B. Termintreue, Durchlaufzeit, nach einem Simulationslauf nicht den Anforderungen entsprechen, lassen sich unterschiedliche Prioritätsregeln per Anwahl austauschen. Dies ermöglicht eine erneue Ablaufplanung mit anschließender Überprüfung der Planungsergebnisse am Simulationsmodell. Zusätzlich lassen sich die unterschiedlichen Planungsergebnisse realitätsnah bewerten/vergleichen, ohne dass ein Programmieraufwand entsteht.

\section{Evaluierung der Ergebnisse}

Mittels der Simulation können die unterschiedlichen Prioritätsregelverfahren miteinander verglichen werden. Hierzu sind die Zielgrößen mittels einheitlicher Kennzahlen zu quantifizieren, um so Verbesserungsansätze zu vergleichen und die jeweilige Regel zu evaluieren.

Im Kontext der Produktionsplanung liegt der Schwerpunkt hierzu auf einer optimalen Termintreue. Die Termineinhaltung lässt sich u.a. durch nachstehende Zielgrößen messen: [18]

Termintreue (\%)

- Pünktliche Aufträge / Gesamtanzahl an Aufträgen

- Ziel $\rightarrow$ Maximierung

Gesamtverspätung (Zeiteinheiten, ZE)

- Ziel $\rightarrow$ Minimierung
Durchschnittliche Verspätung pro Auftrag (ZE/Stk.)

- Gesamtverspätung / Anzahl verspäteter Aufträge

- Ziel $\rightarrow$ Minimierung

Maximale Terminabweichung je Auftrag (ZE/Stk.)

- Ziel $\rightarrow$ Minimierung

Für die Eruierung bestehender Wirkungszusammenhänge (z.B. Termintreue vs. Kapazitätsauslastung) sind zudem die Auslastung der Maschinen- und Transportkapazitäten über die Zeit (Abbildung 5) sowie die Durchlaufzeit der Aufträge zu betrachten. Eine absolute auf eine Periode bezogene Auslastung gibt keinen ausreichenden Aufschluss. Die Auslastungsanalysen wurden im Simulationssystem programmiert und werden anwendungsspezifisch mittels einer dynamischen Animation dem Produktionsplaner visualisiert.

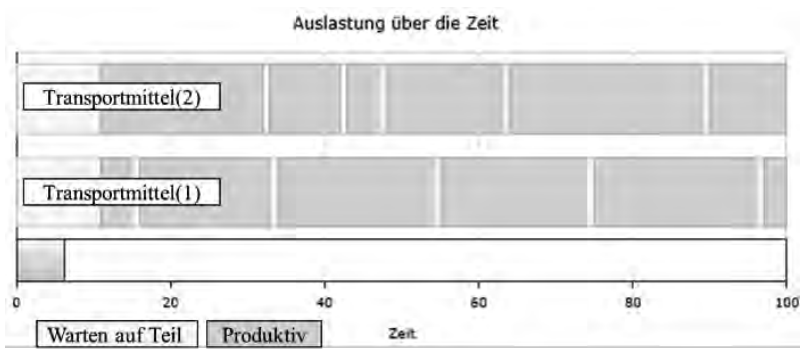

Abbildung 5: Transportmittel-Auslastung über die Zeit

Bedingt durch die Vielzahl an Alternativen einer Matrixfertigung, ist die ausschließliche Anwendung eines PPS-Systems nicht ausreichend [15]. Darüber hinaus muss das Simulationsmodell als DT eines Produktionssystems mit jeder Planungsperiode an die veränderten Ausgangsbedingungen (Auftragsbestand, Terminwünsche, BM-Kapazität, etc.) anpasst werden. Eine Aufwandsarme Erstellung des Simulationsmodells wird mittels der Generierung ermöglicht.

Die „Was-Wäre-Wenn-Szenarien“ erlauben hierbei die Abbildung und Untersuchung von Interdependenzen. So können die Anzahl an Betriebsmitteln, deren Anordnung, eine mengenmäßige Veränderung der Fördersysteme sowie deren Kapazitäten angepasst werden. Dies erlaubt die Darstellung der Zielgrößen in Abhängigkeit einer festzulegenden Prioritätsregel-Kombination und zeigt beispielsweise den Zusammenhang zwischen einer Transportkapazität und der Termintreue auf, welche sich ohne Simulation nicht abbilden lassen.

Wenn die Zielgrößen nicht erreicht wurden, kann eine Neuplanung der Produktion erfolgen. Hierzu können im ersten Schritt die Prioritätsregeln angepasst und ein neuer 
Produktionsablauf, in Abhängigkeit der Produktionstopologie, geplant werden. Diese Planung kann erneut im Simulationsmodell abgesichert werden.

Darüber hinaus bietet das Simulationssystem die Möglichkeit mittels des sogenannten Experimenter einen Planungsablauf zu untersuchen, bis die vorab festgelegten Zielgrößen mit geringster Abweichung erreicht werden (How-to-achieve). Hierbei werden alle Alternativen, welche sich durch das vorhandene Portfolio an hinterlegten Prioritätsregeln ergeben, automatisch durch das System abgebildet. Die benötige Zeit für manuelle Simulationsversuche durch den Produktionsplaner entfallen hierbei.

Verschiedene Zielgrößen wie die DLZ, die Anzahl an verspäteten Aufträgen, der Zeitanteil der durchschnittlichen Verspätung sowie die min. und max. Verspätung werden betrachtet. Die Ergebnisse lassen sich via Schnittstelle (Excel) exportieren und innerhalb einer Graphik abbilden (Abbildung 6).

Dies bietet eine höchstmögliche Transparenz und erlaubt dem Produktionsplaner eine Evaluierung, ob zusätzliche BM-Kapazitäten oder FTS-Kapazitäten eine Verbesserung bringen.

Das erstellte System bietet somit eine schnelle Bestimmung der effizientesten Prioritätsregeln, in Abhängigkeit der jeweiligen Zielgrößen, für eine gegebene Auftragssituation.

\section{Zusammenfassung und Ausblick}

Die automatische Generierung von Simulationsmodellen einer Matrixproduktion erlauben es den Produktionsplaner situationsabhängig ihre Produktionsplanung aufwandsarm abzusichern.

Mittels vorgefertigter Module (Betriebsmittel, Puffer, FTS) sowie unterschiedlicher Prioritätsregeln lassen sich diese mittels einer VBA Schnittstelle parametrisieren, anordnen und erlauben anschließend die Generierung von ablauffähigen Simulationsmodellen ohne Programmierung.

An dem generierten und lauffähigen Simulationsmodell können die dynamischen Produktionseinflüsse untersucht und entsprechende Optimierungsalternativen, anhand von produktionsspezifischen KPIs, evaluiert werden. Darüber hinaus bietet das System die Option alle Ablaufalternativen, die durch unterschiedliche Prioritätsregeln möglich sind, automatisch in einem Simulationslauf zu betrachten und die Zielgrößen graphisch aufbereitet abzubilden. Dies ermöglicht eine effiziente Auswahl der geeignetsten Prioritätsregeln, für die aktuelle Situation in der Produktion.

In einer Weiterentwicklung wird die Verknüpfung mit einem MES-System angestrebt, sodass anlagen- und

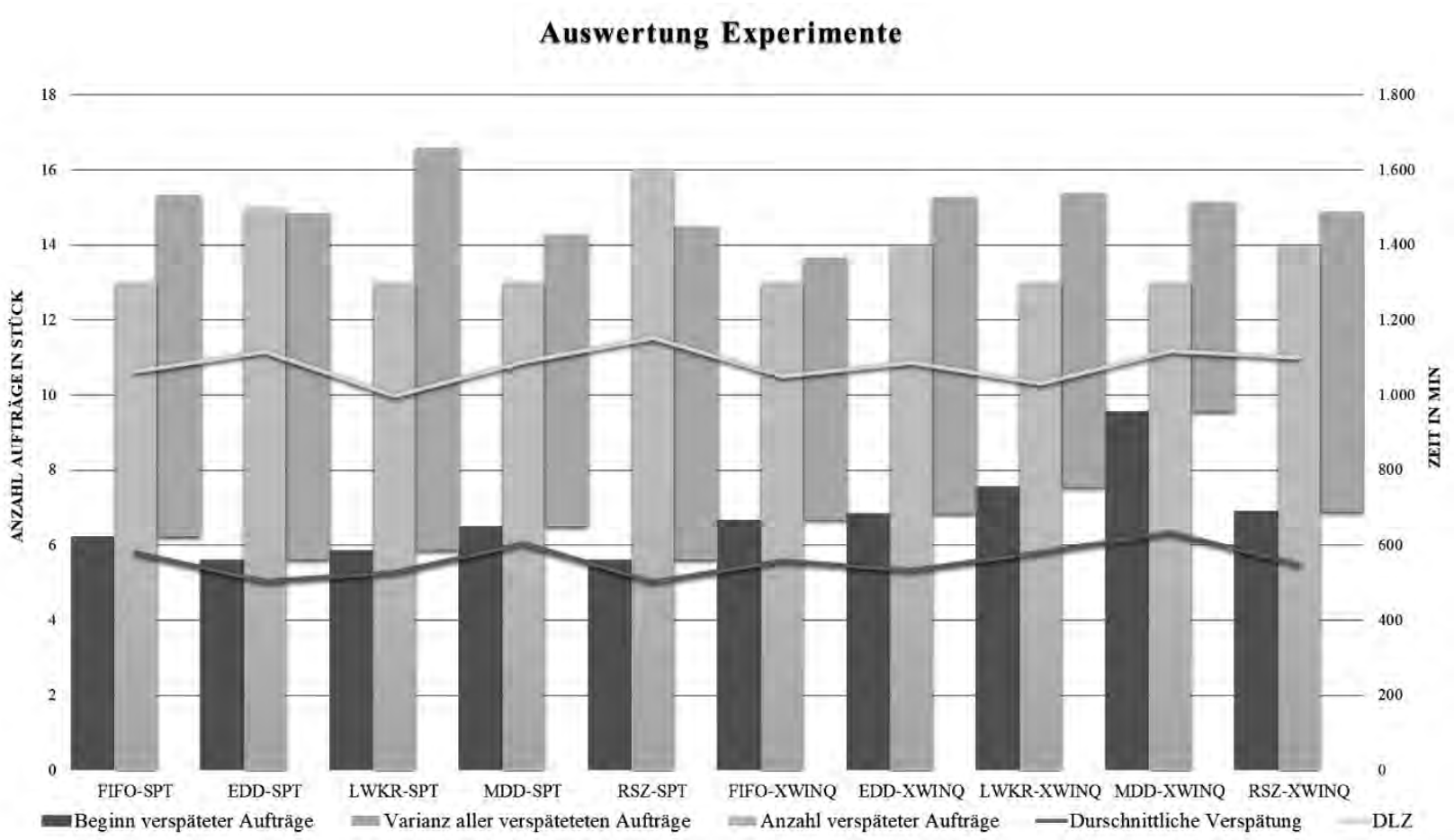

Abbildung 6: Ergebnissdarstellung in Excel 
produktspezifische Kenngrößen bzgl. dem Ausfallverhalten, Rüstzeiten, Qualitätskennzahlen, etc., sowie die damit verbundenen Einflüsse, übernommen und realitätsnah abgebildet werden können. Eine manuelle Parametrierung via VBA-Schnittstelle kann somit weitgehend entfallen.

Darüber hinaus erfolgt eine Erprobung im industriellen Umfeld, um die Funktionalität abzusichern sowie die Überprüfung weiterer Prioritätsregeln zu ermöglichen.

\section{References}

[1] Staufen AG, Institut PTW der technischen Universitä1 Darmstadt. 25 Jahre Lean Management. Köngen: Staufen AG; 2016.

[2] Bauernhansl $\mathrm{T}$, ten Hompel M, Vogel-Heuser B. Industrie 4.0 in Produktion, Automatisierung und Logistik. Wiesbaden: Springer; 2014.

[3] VDI-Richtlinie 3633: Simulation von Logistik-, Materialfluss- und Produktionssystemen, Grundlagen Blatt 1. Düsseldorf: Beuth; 2014.

[4] Bergmann S, Starßburger S, Schulze T. Automatische Generierung adaptiver Modelle zur Simulation von Produktionssystemen. Ilmenau: Univ.-Verl. Ilmenau; 2013.

[5] Baier J, Krieg R. Automatisierter Modellaufbau für Materialflusssimulation in der Nutzfahrzeugproduktion. In Advances in Simulation for Production and Logistics Applications, Stuttgart: Frauenhofer IRB Verlag; 2008. pp. 51-60.

[6] Greschke P, Schönemann M, Thiede S, Herrmann C. Matrix Structures for High Volumes and Flexibility in Production Systems. In Variety Management in Manufacturing. Proceedings of the 47th CIRP Conference on Manufacturing Systems. Procedia CIRP; 2014. pp. 160-165.

[7] KUKA AG. Matrix-Produktion live im KUKA SmartProduction Center. 21 Februar 2018. [Online]. Available: https://www.kuka.com/de-de/presse/news/2 018/02/smartproductioncenter. [Zugriff am 11. September 2020].

[8] AUDI AG. Die Modulare Montage, 17 November 2016. [Online]. Available: https://www.audi-mediacenter.com /de/audi-techday-smart-factory-7076/die-modularemontage-7078. [Zugriff am 11. September 2020].

[9] Popp J, und Wehking K.H. Neuartige Produktionslogistik für eine wandelbare und flexible Automobilproduktion. Logistics Journal: Proceedings; 2016.
[10] Zhang T, Xie S, Rose O. Flexible Job-shop Scheduling with Dynamic Stochastic Machine Sets. In Simulation in Production and Logistics. Dortmund: Fraunhofer Verlag; 2015. pp. 21-28.

[11] Jaehn F, Pesch E. Ablaufplanung. Einführung in Scheduling. Berlin: Springer Verlag; 2014.

[12] Hees A. F. System zur Produktionsplanung für rekonfigurierbare Produktionssysteme. München: Technische Universität München; 2017.

[13] Gutenschwager, Rabe M, Spieckermann S, Wenzel S. Simulation in Produktion und Logistik. Berlin: Springer; 2017.

[14] Briskorn D, Hartmann S. Anwendungen des ResourceConstrained Project Scheduling Problem in der Produktionsplanung. In Produktionsplanung und steuerung. Berlin: Springer; 2015. pp. 109-129.

[15] Niehues M. R. Adaptive Produktionssteuerung für Werkstattfertigungssysteme durch fertigungsbegleitende Reihenfolgebildung. München: Technische Universität München; 2016.

[16] Hotz I. Simulationsbasierte Frühwarnsysteme zur Unterstützung der operativen Produktionssteuerung und -planung in der Automobilindustrie. Magdeburg: Universität Magdeburg; 2007.

[17] Bergmann S. Automatische Generierung adaptiver und lernfähiger Modelle zur Simulation von Produktionssystemen. In Tagungsband zum Doctoral Consortium der WI. Bayreuth: Universität Bayreuth; 2011. pp. 9-16.

[18] Kletti J, Schumacher J. Die perfekte Produktion. Manufacturing Excellence durch Short Interval Technology (SIT). Berlin: Springer; 2014. 